\title{
An asteroseismic Main Sequence model for the $\delta$ Scuti star 44 Tau
}

\author{
R. Garrido, ${ }^{1}$ J. C. Suárez, ${ }^{1,2}$ A. Grigahcène, ${ }^{3}$ M. A. Dupret, ${ }^{2}$ A. Moya ${ }^{1}$ \\ ${ }^{1}$ Instituto de Astrofísica de Andalucía (CSIC), Granada, Spain \\ ${ }^{2}$ Observatoire de Paris-Meudon, France \\ ${ }^{3}$ Observatoire d'Alger, CRAAG, Algeria
}

Antoci et al. (2007) have found that 44 Tau oscillates with 13 independent frequencies with amplitudes higher than the observational limit of $0.6 \mathrm{mmag}$. The observations were made in the two Strömgren filters $(v, y)$ which allow good discrimination of the spherical harmonic $\ell$ of the corresponding non-radial mode, as it was shown by Garrido et al. (1990).

The star has $T_{\text {eff }}=6900 \pm 150 \mathrm{~K} ; \log g=3.6 \pm 0.05\left(\log L / L_{\odot}=1.3 \pm 0.2\right)$ and so$\operatorname{lar}([\mathrm{M} / \mathrm{H}]=0.0 \pm 0.1)$ composition. Different diagnostic diagrams for amplitude ratios and phase differences have been calculated using the "time-dependent convection (TDC)" described by Grigahcène et al. (2005) and Dupret et al. (2004) together with atmospheric parameters taken from Heiter et al. (2002) and Barban et al. (2003). All of these point to the tentative modal identification depicted in Table 1.

The ratio $f_{1} / f_{5}=0.7698 \pm 0.0005$ indicates that these two frequencies correspond to the radial fundamental and first overtone. We can then use the Petersen diagrams to fix the evolutionary status of 44 Tau. Rotation is very low and has not been taken into account (see Suárez et al. 2006). Models with $1.85 M_{\odot}$ and solar composition do not fit the frequency ratio at the required period. We have tried different masses, compositions and overshooting parameters and the only reasonable combinations allow masses from 1.90 to $2.00 \mathrm{M}_{\odot}$ and main sequence models (i.e. hydrogen not yet exhausted in the core).

Work is in progress to find the best model (in a least squares sense) but the closest theoretical frequencies to those observed which agree with the mode identification indicated in Table 1 are for a model with $1.94 M_{\odot}, \alpha_{\mathrm{ov}}=0.3,[\mathrm{M} / \mathrm{H}]=0.10$ and an age of $1130 \mathrm{Myr}$ where the "avoiding crossing" phenomenon is present.

The predicted range of instability depends on the $\alpha$ parameter: low values represent better the excited range of this star when time dependent convection theory is used.

In conclusion, the observed frequencies of 44 Tau can be theoretically explained by a Main Sequence model of $1.94 M_{\odot}$ with a small metal overabundance and a moderate overshooting at an effective temperature $150 \mathrm{~K}$ lower than indicated by the photometric calibration. All the proposed frequencies fit the following constraints: colour identification, instability range, photometric visibility and period ratios for radial modes (Petersen diagrams).

Besides, if our identification is correct, then $\ell$ values up to 2 are visible in this star, at the above mentioned photometric precision, and then three more new frequencies are predicted to be unstable in the observed range which could be detectable from new ground based observations.

Acknowledgments. We would like to thank Alosha Pamyatnykh for valuable discussions during the conference. This work has been supported by the PNE project ESP2004-083855C03-C01. 
Table 1: Observed frequencies, amplitude ratios, phase differences and identifications of the 13 pulsation modes of $44 \mathrm{Tau}$.

\begin{tabular}{cccccc}
\hline \hline Freq & $\nu(\mathrm{c} / \mathrm{d})$ & $\mathrm{v} / \mathrm{y}$ & $\mathrm{v}-\mathrm{y}$ (degrees) & $\ell$ & Identification \\
\hline$f_{1}$ & 6.8980 & $1.459 \pm .006$ & $3.2 \pm 0.3$ & 0 & $\mathrm{~F}$ \\
$f_{2}$ & 7.0060 & $1.45 \pm .01$ & $-1.8 \pm 0.6$ & 1 & $\mathrm{~g} 4$ \\
$f_{3}$ & 9.1175 & $1.46 \pm .01$ & $-1.9 \pm 0.5$ & 1 & $\mathrm{~g} 2$ \\
$f_{4}$ & 11.5196 & $1.43 \pm .01$ & $-2.0 \pm 0.6$ & 1 & $\mathrm{f} 1$ \\
$f_{5}$ & 8.9606 & $1.44 \pm .01$ & $1.5 \pm 0.7$ & 0 & $1 \mathrm{H}$ \\
$f_{6}$ & 9.5613 & $1.46 \pm .01$ & $-0.8 \pm 0.5$ & 1 & $\mathrm{~g} 1$ \\
$f_{7}$ & 7.3034 & $1.48 \pm .03$ & $-9.5 \pm 1.6$ & 2 & $\mathrm{~g} 4$ \\
$f_{8}$ & 6.7953 & $1.41 \pm .06$ & $-9.5 \pm 2.2$ & 2 & $\mathrm{~g} 5$ \\
$f_{9}$ & 9.5801 & $1.49 \pm .1$ & $-8 \pm 3$ & 2 & $\mathrm{~g} 2$ \\
$f_{10}$ & 6.3390 & $1.35 \pm .1$ & $-8 \pm 3$ & 2 & $\mathrm{~g} 6$ \\
$f_{11}$ & 8.6394 & $1.34 \pm .1$ & $-4 \pm 5$ & 1 or 2 & $\mathrm{~g} 3(\ell=2)$ \\
$f_{12}$ & 11.2946 & $1.58 \pm .5$ & $2 \pm 8$ & any & $2 \mathrm{H}$ \\
$f_{13}$ & 12.6967 & $1.48 \pm .9$ & $14 \pm 14$ & any & $\mathrm{f}(\ell=2)$ \\
\hline
\end{tabular}

\section{References}

Antoci V., Breger M., Rodler F., Bischof K., Garrido R., 2007, A\&A, 463, 225

Barban C., Goupil M.-J., Van't Veer-Menneret C., et al., 2003, A\&A, 405, 1095

Dupret M.-A., Grigahcène A., Garrido R., Gabriel M., Scuflaire R., 2004, A\&A, 414, L17

Garrido R., García-Lobo E., Rodríguez E., 1990, A\&A, 234, 262

Grigahcène A., Dupret M.-A., Gabriel M., Garrido R., Scuflaire R., 2005, A\&A, 4341055

Heiter U., Kupka F., van't Veer-Menneret C., et al., 2002, A\&A, 392, 619

Suárez J. C., Garrido R., Goupil M.-J., 2006, A\&A, 447, 649 